\title{
Navegando hacia un modelo de Fast-Track Surgery
}

\author{
Navigating towards a model of Fast-Track Surgery
}

Desde hace algunas décadas hemos venido observando que muchos procedimientos quirúrgicos se realizan, y son cada vez más numerosos, en unidades de cirugía ambulatoria, o al menos, con un tiempo muy limitado de hospitalización. En los comienzos, esta conducta se aplicó sólo a procedimientos menores, en grupos seleccionados de pacientes, pero gradualmente la lista ha crecido de año en año. Todo ello, teniendo en cuenta que en todos los tiempos, se mantienen como los principales objetivos de la cirugía, la eficacia unida a la seguridad. ¿Por qué ha ocurrido ello? No podemos dejar de reconocer que muchos de estos cambios han sido fruto de una estrecha interacción al interior de los sistemas de salud, entre aseguradores y prestadores, pero también está la consideración cada vez más significativa, de la satisfacción del paciente, usuario del sistema. Además, a la cirugía se le exigirá con más fuerza, más calidad y también más equilibrio en los costos. Si pensamos de modo futurista nos imaginamos a pacientes que ingresan por la mañana a un centro determinado y se le practica la terapia quirúrgica programada, aún mayor, usualmente por técnicas mínimamente invasivas; pacientes que regresan a su entorno lo antes posible, muchas veces en el mismo día, con adecuadas instrucciones que implican un buen manejo del dolor.

Cuando medimos el resultado final de la cirugía, habitualmente nos detenemos a analizar la morbilidad, la mortalidad, la estada hospitalaria, el periodo total de reposo antes de volver al trabajo o a la actividad habitual previa, entre otros indicadores. Pues bien, estos parámetros se relacionan con la técnica quirúrgica, con el volumen de pacientes atendidos en un determinado hospital para un procedimiento específico y a los procesos que utiliza cada institución y que determinan su propia cultura. Todo ello está modificándose de modo vertiginoso. Como dijimos, muchos de los procedimientos hoy se efectúan por la vía de la cirugía mínimamente invasiva y, por otro lado, la derivación de pacientes (o la regionalización en otros casos), ha hecho que ciertos centros adquieran más experiencia y seguridad en sus prácticas, obteniendo mejores resultados.

Frente a lo previamente expuesto queda, sin embargo, un amplio espacio para acelerar otro tanto la recuperación postoperatoria y disminuir el período de convalecencia. Y aún más, disminuir las complicaciones. Desde hace poco más de una década ha venido madurando la puesta en escena de un programa dirigido hacia esos objetivos, programa que compromete la interacción de varios profesionales y especialistas. Es lo que se ha dado por conocer como Fast-Track Surgery (FTS) o Recuperación Postquirúrgica Mejorada (Enhanced Recovery After Surgery, ERAS). Según lo propuesto por Kehlet ${ }^{1,2}$, quien ha explorado este tema en profundidad, se trata de un programa multimodal, en que participan cirujanos, anestesiólogos, kinesiólogos y enfermeras, con el objetivo de que este dirigido trabajo en equipo mejore la recuperación del paciente quirúrgico y reduzca las complicaciones.

Se han identificado tres componentes fundamentales de los programas de Fast-Track Surgery, y que le han otorgado la sustentación. Ellos son: los principios de la medicina basada en la evidencia, la reducción del estrés quirúrgico y el manejo del dolor. Estos afectan el curso del paciente y su entorno desde el período preoperatorio, durante el intraoperatorio y por toda su evolución posterior.

Si nos detenemos en el primero de los puntos, hoy es aplastante la evidencia que ha echado por tierra muchos de los mitos de la cirugía, que se habian perpetuado por décadas. La utilización de drenajes intraperitoneales y de sonda nasogástrica de modo rutinario y el rol de la preparación mecánica del colon, cuya inefectividad hoy está probadamente demostrada, son algunos de los ejemplos que han visto acabar su indicación para la gran mayoría de los casos. Debemos agregar la evidencia positiva para implementar un programa estandarizado de profilaxis antibiótica y de tromboprofilaxis como medidas que efectivamente disminuyen la morbilidad. La adecuada instrucción del paciente sobre su condición, de modo que conozca y asuma responsablemente su decisión, se ha demostrado como una medida eficiente para reducir las complicaciones y cursar un mejor postoperatorio. También está probado el efecto beneficioso de optimizar en el preoperatorio las condiciones de disfunción orgánica previa, como enfermedad cardíaca, diabetes mellitus 
EDITORIAL

y enfermedad pulmonar obstructiva. Este capitulo también comprende la evidencia sobre la capacidad e inocuidad de tolerar dieta desde las primeras horas del postoperatorio, aún en presencia de ciertas anastomosis del tubo digestivo.

En relación a la reducción del estrés quirúrgico, mediado muchas veces por una respuesta endocrinometabólica, se apunta a reducir el catabolismo, una alta demanda cardiaca y una exagerada respuesta inflamatoria, manteniendo la capacidad inmune y de defensa contra las infecciones, a la vez que una adecuada capacidad de cicatrización de los tejidos, como lo ha puntualizado el mismo autor ${ }^{3}$. En este sentido, la cirugía mínimamente invasiva ha jugado, y lo seguirá haciendo, un rol determinante en la mejoría de la evolución postoperatoria. Hoy es indiscutible que esta modalidad quirúrgica se asocia a menos dolor, estadas hospitalarias más breves y menor morbilidad. En el cuidado perioperatorio el rol del anestesiólogo es, también, relevante; le corresponde el adecuado manejo del volumen (para no encharcar a los enfermos), y el control de la temperatura corporal (para evitar la hipotermia), entre otros aspectos.

El tercer pilar de un programa de FTS apunta a escoger la anestesia y analgesia postoperatoria más adecuada para cada paciente en particular, bajo la premisa de que la utilización de una analgesia multimodal libre de opiodes, facilita la movilidad precoz del paciente y disminuye la respuesta catabólica.

Todos estos conceptos han sido aplicados progresivamente en diversas cirugías; inicialmente en cirugía colónica, hoy ampliado a una gama de procedimientos: herniorrafias, resecciones pulmonares, endarterectomía de carótida, prostatectomía, nefrectomía, adrenalectomía, e incluso resecciones mayores de hígado y de páncreas, como lo demuestran series recientes, reduciendo significativamente los tiempos de hospitalización y la tasa de complicaciones ${ }^{4,5}$.

Por cierto que la gran inquietud para el cirujano tradicional es que esta forma de hacer cirugía implique mayores índices de complicaciones y, por lo mismo, de reingreso al hospital, pero los estudios, categóricamente, no han demostrado tal suposición. Tampoco ocurre que un protocolo de FTS aumente los costos totales, pues pudieran observarse menores costos iniciales por la disminución de días intrahospitalarios, pero un aumento de los costos posteriores, por la necesidad de cuidados especiales fuera del hospital, aunque faltan más estudios en ese sentido.

En suma, el desarrollo quirúrgico navega en el sentido que aquí se ha esbozado: cirugías muy seguras, con reducidos tiempos de hospitalización y de recuperación y costos ajustados. Corresponde preparar a los equipos para que ello se haga de la forma más adecuada posible, pues estamos conscientes que hay resistencia a cambios de este tipo. De hecho, la adhesión mundial a los programas de FTS ha sido lenta. En lo actual, es ir dando al timón las correcciones necesarias con el fin de llegar al puerto seguro, sin dilaciones y con toda la tripulación.

Dr. Ricardo Espinoza $G$. Departamento de Cirugía Facultad de Medicina. Universidad de los Andes Santiago, Chile.

\section{Referencias}

1. Kehlet H, Dahl JB. Anesthaesia, surgery, and challenges in postoperative recovery. Lancet 2003; 362: 1921-1928.

2. Kehlet H. Multimodal approach to postoperative recovery. Curr Opin Crit Care 2009; 15: 355-358.

3. Kehlet H, Wilmore DW. Evidence-Based Surgical Care and the Evolution of Fast- Track Surgery. Ann Surg 2009; 248 : 189-198.

4. Berberat PO, Ingold H, Gulbinas A, Kleeff J. Fast track- different implications in pancreatic surgery. J Gastrointest Surg 2007; 11: 880-887.

5. Van Dam RM, Hendry PO, Coolsen MME, Bemelmans MHA. Initial experience with a multimodal enhanced recovery programme in patients undergoing liver resection. Br J Surg 2008; 95: 969-975. 